\title{
Propuesta de una escala para medir la calidad del servicio de los centros de atención secundaria de salud*
}

\author{
Eduardo Torres Moraga** \\ Jorge Lastra Torres***
}

Sumario: 1. Introducción; 2. La calidad del servicio en el sector de la salud; 3. Metodología y validez de contenido; 4. Análisis de la escala de medida y resultados; 5. Discusión.

Summary: 1. Introduction; 2. Quality of service in the healthcare sector; 3. Methodology and content validity; 4. Analysis of the scale of measure and results; 5. Discussion.

Palabras clave: calidad del servicio; salud pública; satisfacción del paciente; fiabilidad; validez de escalas.

KEY WORDS: service quality; public health; patient satisfaction; reliability; validity of scales.

El Ministerio de Salud en Chile se ha planteado como uno de sus principales objetivos, proveer servicios acorde a las expectativas de la población. Para lograrlo, se requiere necesariamente, conocer cuál es la calidad del servicio ofrecida por los centros de salud. En Chile, los centros de atención secundaria, no han desarrollado escalas de calidad del servicio que tengan un buen grado de validez, fiabilidad y dimensionalidad. Este artículo, propone una escala que cumpla estas condiciones, para medir de manera óptima la calidad del servicio que los Centros de Atención Secundaria

\footnotetext{
* Artículo recibido en abr. 2008 e acepto en jun. 2008.

** Académico e investigador del Departamento de Administración, Facultad de Economía y Negocios, Universidad de Chile. Director del Magíster en Administración (para profesionales), Universidad de Chile. Doctor en Gestión, Universidad de Zaragoza, España. Dirección: Diagonal Paraguay 257, of. 1103, Santiago, Chile. Correo electrónico: eduardot@fen.uchile.cl.

$* * *$ Director del Servicio de Salud Metropolitano Central, Chile. Médico cirujano, Universidad de Chile, Chile. Magíster en Estudios Sociales y Políticos, Universidad Alberto Hurtado, Chile. Dirección: Victoria Subercaseaux 381, Santiago, Chile. Correo electrónico: jorge.lastra@redsalud. gov.cl.
} 
ofrecen a sus pacientes. Para lograr este objetivo, se aplicó una encuesta estructurada y posteriormente se realizó un exhaustivo análisis psicométrico de los datos, a través de una serie de análisis exploratorios y confirmatorios que incluyó ecuaciones estructurales. Este estudio permitió construir y proponer una escala fiable, válida y con buen grado de dimensionalidad, compuesta por cinco subescalas claramente relacionadas e integradas en un único constructo. Estas son: confiabilidad, empatía, capacidad de respuesta, accesibilidad y tangibles.

\section{Proposal of a scale to measure the quality of service in secondary healthcare centers}

Chile's Ministry of Health has made it one of its main objectives to provide services that meet the expectations of the population. To achieve this, it is necessary to know what the quality of service offered by healthcare centers is like. In Chile, secondary healthcare centers have not developed quality of service scales that have a good level of validity, dependability, and dimensionality. This article proposes a scale that fulfills these conditions so as to measure in an optimal way the quality of service that secondary healthcare centers offer their patients. To achieve this objective, a structured survey was applied, followed by an exhaustive psychometric analysis of the facts through exploratory and confirmatory analysis that included structural equations. This study allowed for the construction and proposal of a scale that is valid, dependable and has a good level of dimensionality composed of five subscales that are clearly related and integrated in one single construct. These are: reliability, empathy, response capacity, accessibility, and tangibility.

\section{Introducción}

En el sector de la salud, la calidad del servicio ha cobrado un especial protagonismo. De hecho, el Ministerio de Salud en Chile, ha incorporado dentro de los objetivos principales de su gestión, la promoción de una atención de salud comprometida, acogedora y participativa. Esta iniciativa, sumado el hecho, que los pacientes son cada vez más exigentes, ha motivado a las instituciones de salud pública, a implementar estrategias para ofrecer un mejor servicio a sus pacientes, basado en las expectativas y en una gestión más eficiente de cada uno de los factores que forman parte de la calidad del servicio.

Entre estas instituciones, se encuentran los centros de atención secundaria de salud, que son los encargados de ofrecer programas y prestaciones de salud especializados a los usuarios. Para que los pacientes sean atendidos por un médico especialista en la atención secundaría, éstos deben ser derivados por medio de una interconsulta, que el médico del consultorio o centro de salud primario emite al centro de atención secundaria. 
A pesar de la importancia que tiene esta iniciativa, los centros de atención secundaria de salud, aún no han desarrollado escalas válidas, fiables y dimensionales que permitan medir los factores claves de la calidad del servicio que ofrecen a sus pacientes. De hecho, actualmente no tienen una escala de medida que cumpla estas condiciones esenciales, ya sea para tomar decisiones respecto a la situación actual del centro (en cuanto al proceso de atención al paciente), así como para gestionar los elementos claves que permitan lograr su posterior satisfacción.

Conscientes de este desafío, el presente articulo, tiene como objetivo proponer una escala con suficiente grado de fiabilidad, validez y dimensionalidad, que permita medir la "Calidad del Servicio desde la perspectiva de los pacientes de los Centros de Atención Secundaria" de salud (CCAS). Poniendo énfasis en los procesos de atención que el centro ofrece a sus pacientes y no en los resultados clínicos de la atención del médico, que en este último caso, necesitan de otro instrumento de evaluación.

A través de esta escala de medida, cada centro, no sólo podrá conocer la calidad de los servicios que ofrece, sino también, compararla con la de otros servicios similares, y tomar las acciones necesarias para mejorar su gestión y en consecuencia la satisfacción de sus pacientes actuales y potenciales.

\section{La calidad del servicio en el sector de la salud}

En relación a los estudios que han medido la calidad del servicio en sector de la salud, podemos mencionar, el realizado por Nelson et al. (1989). Estos autores, por medio de un sistema de juicio del paciente, desarrollaron un estudio en el Hospital Corporation of America. Éste dio como resultado, un cuestionario con once dimensiones de calidad que incluyó 68 ítems de medida.

Posteriormente, Donabediam (1990) propuso que la calidad de la atención, debe basar su medición en tres componentes: la atención técnica, el manejo de la relación interpersonal y el ambiente en el que se lleva a cabo el proceso de atención.

Esa misma década Bowers, Swan y Koehler (1994) a partir de la escala Servqual, desarrollaron un instrumento específico para medir la calidad del servicio en dos contextos de atención médica: la asistencia médica y el cuidado médico. Este instrumento consideró seis dimensiones en la medición de la calidad del servicio (información, fiabilidad, capacidad de respuesta, accesibilidad, empatía y cuidados). 
A fines de esa década, Jun, Peterson y Zsidisin (1998) identificaron 11 dimensiones que definen la calidad del cuidado del paciente y su satisfacción, tanto desde la perspectiva médica como desde el punto de vista de la administración (tangibilidad, fiabilidad, capacidad de respuesta, competencia, cortesía, información, accesibilidad, cuidados, resultados, empatía y continuidad).

Posteriormente, a partir de la escala Servqual, Gea et al. (2001) desarrollaron el instrumento Sercal, para medir la calidad del servicio de un centro médico-quirúrgico perteneciente a un hospital. Este instrumento, incluyó las dimensiones, accesibilidad al servicio, confort, personalización, garantía y fidelidad.

Ese mismo año, Mira et al. (2001) presentaron la escala Servqhos de calidad de la asistencia hospitalaria percibida por el paciente. Esta escala fue aplicada a dos hospitales públicos a lo largo de un período superior a dos años. Esta escala medió los factores amabilidad, capacidad de respuesta, confianza en los profesionales, trato de enfermería, tiempo de espera, estado de la habitación, informaciones, capacidad de resolver los problemas, puntualidad, rapidez de respuesta, interés, facilidad para acudir al centro, preparación profesional, tecnología biomédica y trato al paciente.

$\mathrm{Al}$ año siguiente De Man et al. (2002) presentaron un estudio en que analizan la importancia de diversas dimensiones de la escala Servqual y su relación con la satisfacción del paciente en el contexto de la medicina nuclear. Algunas dimensiones de esta escala no fueron confirmadas en este nuevo contexto. De hecho, los pacientes consideraron los bienes materiales y la seguridad como una sola dimensión, y además la dimensión original empatía fue dividida en empatía y conveniencia.

Por último, Chang et al. (2006) realizaron un estudio para determinar como los encuentros del servicio afectan la satisfacción de los pacientes. Identificaron que los que en mayor medida afectan la satisfacción con los tratamientos médicos, son los encuentros del paciente con: los profesionales médicos, los profesionales encargados del cuidado, con el personal de servicio, $\mathrm{y}$ con los espacios e instalaciones.

\section{Metodología y validez de contenido}

Para garantizar una escala con suficiente grado de validez de contenido (Deng y Dart, 1994), se consideró como punto de partida una serie de estudios previos (Nathorst-Böös et al., 2001; Maderuelo et al., 1996; Parasuraman, Zeithalm y Berry, 1988; Ware y Snyder, 1975; Lewis, 1994; Nelson et al., 1989; 
McKinley et al., 1997; Jun, Peterson y Zsidisin, 1998; Mira et al., 1999; Chang et al., 2006), los cuales permitieron construir una escala de medida inicial. Posteriormente, se depuró esta escala a través de un exhaustivo análisis recomendado por De Wulf y Odekerken-Schröder (2003). De hecho, se realizaron una serie de entrevistas con pacientes de los centros de atención secundaria de salud de diferentes sectores de la ciudad de Santiago de Chile, y además de entrevistas con directivos de centros de salud, con los cuales se pudo analizar las diferentes dimensiones e indicadores incluidos en el constructo inicial propuesto. Este análisis permitió, sumar o eliminar dimensiones y/o indicadores de tal forma de reflejar más adecuadamente la CCAS. Finalmente, con este cuestionario inicial, se realizó un pre-test cuantitativo a una muestra aleatoria de 50 usuarios de diferentes centros de atención secundaria de salud. Posteriormente con esos datos, se realizó un análisis factorial exploratorio y se calculó el alpha de Cronbach a cada una de las dimensiones resultantes. Con este análisis previo, se pudo confirmar la existencia de cada una de las dimensiones que resultaron de las entrevistas realizadas previamente. Por lo tanto, la escala utilizada en el cuestionario definitivo quedó compuesta por 29 ítems agrupados en las dimensiones confiabilidad, empatía, capacidad de respuesta, accesibilidad y tangibles (ver anexo).

Todos los ítems o indicadores fueron redactados como afirmaciones, donde los encuestados debían responder a través de una escala Likert de 1 (totalmente en desacuerdo) a 7 (totalmente de acuerdo). Se aplicó una encuesta estructurada, en forma personal dentro de los dos centros secundarios de salud, que se encuentran entre los más representativos de Santiago de Chile, ya que atienden a pacientes de sectores muy diversos de esta ciudad. Estos son, el Centro de Referencia de Salud de Maipú y el Centro de Diagnósticos y Terapéuticos del Hospital San Borja Arriarán. Después de una prueba de campo, que arrojó como resultado, que los pacientes después de ser inmediatamente atendidos por el médico mostraban sesgos en sus respuestas, la encuesta fue aplicada a pacientes en la sala de espera, antes de ser atendidos, y con el requisito que hubieran acudido al mismo centro de salud dentro del último mes. Por lo tanto, la entrevista se centró en evaluar la experiencia anterior del paciente con el servicio. Con esto se aseguró, que cada entrevistado esté en condiciones de evaluar todas las dimensiones e ítems de calidad del servicio considerados en el cuestionario. Las entrevistas se realizaron en todos los horarios en que se atienden pacientes en las consultas. Con este procedimiento, en ambos centros de salud se encuestaron a 500 usuarios procedentes de la mayoría de las comunas de Santiago de Chile (Santiago centro, Maipú, Pedro Aguirre Cerda, Cerrillos, Estación Central, San Ramón, Pudahuel, La 
Pintana, San Joaquín, La Cisterna, La Florida, Renca, Padre Hurtado, Macul y Recoleta). De las 500 encuestas, 498 resultaron válidas. El 44\% de ellas se aplicaron en el Centro de Referencia de Salud de Maipú y el 56\% en el Centro de Diagnósticos y Terapéuticos. El estudio de campo fue realizado entre el 22 de enero y el 2 de febrero del año 2007.

El 68,5\% de los encuestados habían sido derivados al centro de atención secundaría, desde un consultorio de salud, el 12,9\% desde algún servicios de urgencia, el $12 \%$ desde un hospital y el 6,6\% desde otros tipos de centros de salud. El 77,5\% de los encuestados son mujeres. El 6,4\% de los entrevistados tenía entre 17 y 24 años de edad, el 8,5\% entre 25 y 34 años, el 18,2\% entre 35 y 44 años, el $21,3 \%$ entre 45 y 54 años, el 19,9\% entre 55 y 64 años, el $16,5 \%$ entre 65 y 74 años y el 9,2\% tenía más de 74 años de edad. El 28,9\% de los encuestados tenía educación básica, el 58,4\% tenia estudios de educación secundaría, el 7\% tenía estudios en institutos de formación técnica, el 4,7\% tenía estudios universitarios y sólo el $1 \%$ no tenia ningún tipo de estudios formales.

\section{Análisis de la escala de medida y resultados}

Una vez efectuadas las entrevistas, se realizó un análisis psicométrico de los datos. Con la finalidad de obtener un constructo que permita medir la CCAS como una variable latente y que además cumpla con un buen grado de fiabilidad, validez y dimensionalidad, se realizó un estudio de los datos en tres etapas.

\section{Primera etapa: análisis exploratorio}

Fiabilidad inicial y unidimensionalidad de cada una de las subescalas

En este análisis, el estadístico alpha de Cronbach arrojó en todos los casos valores muy superiores a 0,6 , que es el recomendado para un análisis exploratorio (Nunnally, 1978; Hair et al., 1998) (confiabilidad 0,70, empatía 0,87 , capacidad de respuesta 0,73 , accesibilidad 0,73 y tangibles 0,82 ). Sin embargo, la variable ACC5 perteneciente a la dimensión accesibilidad fue eliminada del análisis, por presentar una correlación de 0,25 , que está por debajo de 0,3 que es el mínimo establecido para este tipo de análisis (Nurosis, 1993). 
Los análisis factoriales de componentes principales con rotación varimax arrojaron en todos los casos un buen grado de undimensionalidad (Hair et al., 1998) (tabla 1). Por lo tanto, con este procedimiento no fue necesario eliminar indicadores.

Tabla 1

Análisis factorial exploratorio de las subescalas de CCAS

\begin{tabular}{|c|c|c|c|c|c|c|c|c|c|}
\hline \multicolumn{2}{|c|}{ Confiabilidad } & \multicolumn{2}{|c|}{ Empatía } & \multicolumn{2}{|c|}{$\begin{array}{l}\text { Capacidad de } \\
\text { respuesta }\end{array}$} & \multicolumn{2}{|c|}{ Accesibilidad } & \multicolumn{2}{|c|}{ Tangibles } \\
\hline Indicador & Carga & Indicador & Carga & Indicador & Carga & Indicador & Carga & Indicador & Carga \\
\hline CONF1 & 0,618 & EMP1 & 0,764 & CAP1 & 0,604 & $\mathrm{ACCl}$ & 0,743 & TAN 1 & 0,717 \\
\hline CONF2 & 0,774 & EMP2 & 0,842 & CAP2 & 0,683 & ACC2 & 0,819 & TAN2 & 0,690 \\
\hline CONF3 & 0,548 & EMP3 & 0,843 & CAP3 & 0,705 & $\mathrm{ACC} 3$ & 0,762 & TAN3 & 0,754 \\
\hline CONF4 & 0,583 & EMP4 & 0,862 & CAP4 & 0,735 & ACC4 & 0,839 & TAN4 & 0,826 \\
\hline \multirow[t]{3}{*}{ CONF5 } & 0,726 & EMP5 & 0,567 & CAP5 & 0,776 & & & TAN5 & 0,770 \\
\hline & & EMP6 & 0,656 & & & & & TAN6 & 0,642 \\
\hline & & EMP7 & 0,743 & & & & & TAN7 & 0,519 \\
\hline \multicolumn{2}{|c|}{ Valor propio: 3,52 } & \multicolumn{2}{|c|}{ Valor propio: 4,05 } & \multicolumn{2}{|c|}{ Valor propio: 2,47} & \multicolumn{2}{|c|}{ Valor propio: 2,51} & \multicolumn{2}{|c|}{ Valor propio: 3,52 } \\
\hline \multicolumn{2}{|c|}{$\begin{array}{c}\text { Varianza exp. } \\
50,2 \%\end{array}$} & \multicolumn{2}{|c|}{ Varianza exp. 57,8\% } & \multicolumn{2}{|c|}{$\begin{array}{c}\text { Varianza exp. } \\
49,4 \%\end{array}$} & \multicolumn{2}{|c|}{ Varianza exp. 62,6\% } & \multicolumn{2}{|c|}{ Varianza exp. 50,2\% } \\
\hline
\end{tabular}

\section{Segunda etapa: análisis confirmatorio}

En esta etapa se realizó un análisis más exhaustivo de las escalas de medida representativas de la CCAS. Se llevó a cabo a través de un análisis factorial confirmatorio utilizando el método de ecuaciones estructurales. Aquí se realizó un proceso de mejora mediante una estrategia de desarrollo de modelos (Hair et al., 1998), que consiste en eliminar sucesivamente aquellos indicadores o variables menos adecuadas para lograr un buen ajuste de éste. Esta eliminación se realizó considerando los tres criterios propuestos por Jöreskog y Sörbom (1993). ${ }^{1}$

Con este análisis, fue necesario eliminar los indicadores TAN7, CONF4, CAP1, CONF3, ya que no cumplían con el segundo criterio. Al aplicar el tercer

\footnotetext{
${ }^{1} \mathrm{El}$ primer criterio consiste en apartar del análisis aquellos indicadores que tengan una débil condición de convergencia ( $t$ de Student menor a 2,58. $p=0,01$ ). Un segundo criterio, es eliminar aquellos indicadores que no sean sustanciales ( $\lambda$ menores a 0,5$)$. Por último, se eliminan aquellos indicadores que son menos explicados por el factor que los representa $\left(R^{2}\right.$ menor a 0,3$)$.
} 
criterio se eliminó del estudio la variable EMP1; con lo que se llegó hasta a una tercera versión del modelo (figura 1 ), que es la que representaría el modelo óptimo para medir la CCAS (tabla 2).

\section{Tabla 2}

\section{Sucesivos análisis factoriales confirmatorios al modelo de CCAS}

\begin{tabular}{|c|c|c|c|c|}
\hline Ajuste del modelo & Valor óptimo & Etapa 1 & Etapas 2 & $\begin{array}{c}\text { Etapa } 3 \\
\text { Mejor modelo }\end{array}$ \\
\hline Item eliminados & & & $\begin{array}{l}\text { TAN7, CONF4, } \\
\text { CAP1, CONF3 }\end{array}$ & EMP1 \\
\hline \multicolumn{5}{|c|}{ Medidas de ajuste absoluto } \\
\hline $\begin{array}{l}\chi^{2}(\mathrm{~g} \cdot \mathrm{l} .) \\
\mathrm{p}\end{array}$ & & $\begin{array}{c}1529,51(340) \\
P \leq 0,001\end{array}$ & $\begin{array}{c}1339,143(242) \\
P \leq 0,001\end{array}$ & $\begin{array}{c}1109,162(220) \\
P \leq 0,001\end{array}$ \\
\hline NCP & Menor valor & 1189,51 & 1097,143 & 889,162 \\
\hline SNCP & Menor valor & 2,388 & 2,203 & 1,785 \\
\hline GFI & Mayor valor & 0,802 & 0,794 & 0,822 \\
\hline \multicolumn{5}{|c|}{ Medidas de ajuste incremental } \\
\hline IFI & Mayor valor & 0,818 & 0,819 & 0,845 \\
\hline $\mathrm{CFI}$ & Mayor valor & 0,817 & 0,818 & 0,844 \\
\hline NFI & Mayor valor & 0,778 & 0,788 & 0,813 \\
\hline \multicolumn{5}{|c|}{ Medidas de ajuste de parsimonia } \\
\hline AIC & Menor valor & 849,519 & 855,143 & 669,162 \\
\hline Normed $\chi^{2}$ & {$[1 ; 5]$} & 4,498 & 5,533 & 5,041 \\
\hline
\end{tabular}

Con el objeto de determinar la multidimensionalidad de la variable CCAS, se desarrolló una estrategia de modelos rivales (Steenkamp y Van Trijp, 1991). Este contraste de modelos rivales se llevó a cabo comparando un modelo de primer orden, es decir, uno en el que todos los indicadores o ítems del constructo de CCAS cargan sobre un solo factor; con otro de segundo orden, donde el constructo está representado por diferentes dimensiones. ${ }^{2}$ En el tabla 3 se puede apreciar que el ajuste del modelo de segundo orden, es mejor que el de primer orden, confirmando de esta manera la multidimensionalidad de la variable CCAS.

\footnotetext{
${ }^{2}$ El modelo de primer orden, al igual que el de segundo orden, no consideran para su análisis los indicadores que fueron eliminados durante el proceso de depuración.
} 


\section{Modelo estructural óptimo de CCAS}

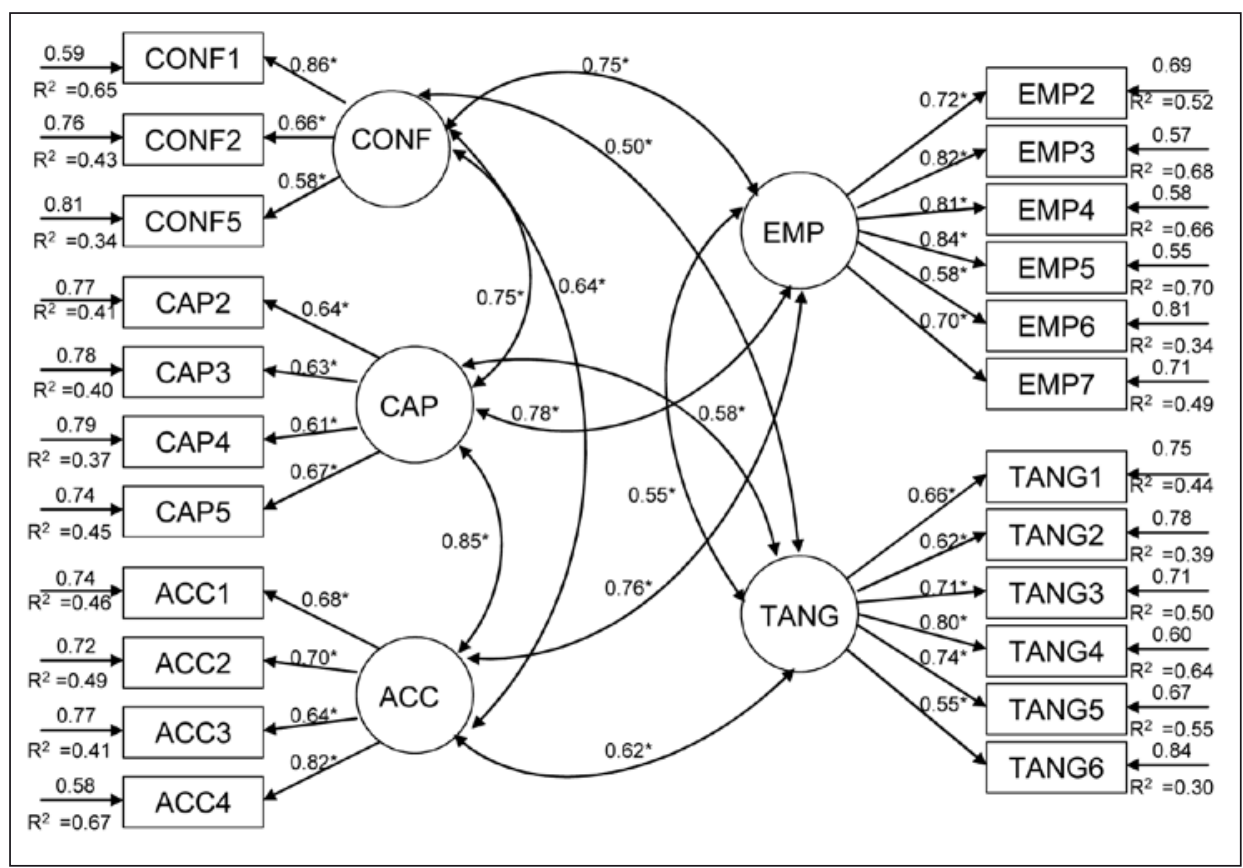

* significativo al 0.01 .

Tabla 3

Análisis mutidimensional del constructo CCAS

\begin{tabular}{|cccc|}
\hline \multirow{2}{*}{ Ajuste del modelo } & \multirow{2}{*}{ Valor óptimo } & \multicolumn{2}{c|}{ Modelos } \\
\cline { 3 - 4 } & & Primer orden & Segundo orden \\
\hline Medidas de ajuste absoluto & $1946,088(230)$ & $1131,236(225)$ \\
$\chi^{2}$ (g.l.) & & $\mathrm{P} \leq 0,001$ & $\mathrm{P} \leq 0,001$ \\
$\mathrm{P}$ & & 1716,088 & 906,236 \\
$\mathrm{NCP}$ & Menor valor & 3,445 & 1,819 \\
SNCP & Menor valor & 0,711 & 0,820 \\
GFI & Mayor valor & & \\
Medidas de ajuste incremental & Mayor valor & 0,700 & 0,841 \\
IFI & Mayor valor & 0,698 & 0,841 \\
CFI & Mayor valor & 0,672 & 0,810 \\
NFI & Menor valor & 1486,088 & 681,236 \\
Medidas de ajuste de parsimonia & 8,46 & 5,02 \\
AIC & {$[1 ; 5]$} &
\end{tabular}




\section{Tercera etapa: análisis de fiabilidad definitiva y validez de la escala de medida}

En esta etapa, se debe verificar el grado de fiabilidad y validez del constructo resultante de los análisis realizados anteriormente. Para determinar la fiabilidad de las subescalas se realizó un análisis del alpha de Cronbach definitivo y se aplicó el coeficiente de fiabilidad compuesta del constructo.

Como podemos apreciar en la tabla 4, los índices de fiabilidad de alpha de Cronbach cumplen en todos los casos con el umbral establecido de 0,7 (la eliminación de algunos indicadores permitieron mejorar los índices de las dimensiones empatía y accesibilidad). Sin embargo, en dos de las dimensiones, los índices arrojados por el análisis de fiabilidad compuesta del constructo, están por debajo de 0,7 superando el nivel de 0,65. A pesar de estos últimos resultados, los buenos índices de alpha de Cronbach permiten garantizar en buen grado la fiabilidad de estas dimensiones.

Tabla 4

Fiabilidad de las escalas del constructo CCAS

\begin{tabular}{|ccccc|}
\hline Confiabilidad & Empatía & $\begin{array}{c}\text { Capacidad de } \\
\text { respuesta }\end{array}$ & Accesibilidad & Tangibles \\
\hline \multirow{5}{*}{0.70} & Alpha de Cronbach \\
& 0,88 & 0,71 & 0,82 \\
0,67 & 0,84 & Fiabilidad de Constructo & 0,80 & 0,79 \\
\hline
\end{tabular}

Para medir la validez del constructo, se siguió el esquema planteado por Nunnally (1978). Según este autor, para que exista validez, debe comprobarse que la escala empleada tenga validez de contenido, validez de constructo y validez con relación a un criterio.

La validez de contenido, está en gran parte garantizada, debido a que las subescalas utilizadas para medir la CCAS se han diseñado a partir de un análisis detallado de la literatura. Posteriormente, han sido sometidas al juicio y a la discusión de expertos, y han sido objeto de un proceso de depuración posterior.

La validez convergente, se confirmó al observar que todos los coeficientes estandarizados resultaron estadísticamente significativos al 0.01 y mayores a 0.5 (Bagozzi y Yi, 1988; Anderson y Gerbing, 1988) (figura). Por su parte, la validez discriminante del modelo propuesto (Bagozzi, 1981) también fue 
confirmado. Como se puede aprecia en la tabla 5, las chi-cuadrado que miden las diferencias entre el modelo teórico resultante del análisis factorial confirmatorio y los diferentes modelos alternativos, son altamente significativas en todos los casos estudiados. Además se puede observar, que en los intervalos de confianza, las correlaciones se alejan considerablemente del valor "1".

Tabla 5

Validez discriminante del constructo CCAS

\begin{tabular}{|lcc|}
\hline Pares de constructor & $\begin{array}{c}\text { Test diferencias } \\
\chi^{2}(\mathrm{~g} . \mathrm{I}) ; \mathrm{p} \text {-value }\end{array}$ & $\begin{array}{c}\text { Intervalos de } \\
\text { confianza }\end{array}$ \\
\hline Confiabilidad-empatía & $75,97(1) ; 0.000$ & 0,$685 ; 0,809$ \\
Confiabilidad-capacidad de respuesta & $40,09(1) ; 0.000$ & 0,$672 ; 0,824$ \\
Confiabilidad-accesibilidad & $108,16(1) ; 0.000$ & 0,$561 ; 0,721$ \\
Confiabilidad-tangibles & $177,56(1) ; 0.000$ & 0,$407 ; 0,587$ \\
Empatía-capacidad de respuesta & $89,71(1) ; 0.000$ & 0,$717 ; 0,837$ \\
Empatía-accesibilidad & $186,74(1) ; 0.000$ & 0,$700 ; 0,812$ \\
Empatía-tangibles & $550,43(1) ; 0.000$ & 0,$475 ; 0,627$ \\
Capacidad de respuesta-accesibilidad & $36,17(1) ; 0.000$ & 0,$794 ; 0,910$ \\
Capacidad de respuesta-tangibles & $194,43(1) ; 0.000$ & 0,$498 ; 0,660$ \\
Accesibilidad-tangibles & $278,32(1) ; 0.000$ & 0,$550 ; 0,698$ \\
\hline
\end{tabular}

Finalmente, el análisis de validez con relación a un criterio, se comprobó al observar que en efecto, la CCAS se relaciona con alguna variable criterio que la teoría indica. En este caso, su correlación con los diferentes indicadores que miden el manejo de los factores situacionales en un centro de salud de atención secundaria, resultó altamente significativa (tabla 6).

Tabla 6

Relación entre CCAS y los factores situacionales

\begin{tabular}{|c|c|}
\hline Variables factores situacionales & $r$ \\
\hline $\begin{array}{l}\text { Si hay una situación extraordinaria (corte de luz, de agua etc.) en el centro me informan } \\
\text { oportunamente lo que sucede }\end{array}$ & $0,355^{*}$ \\
\hline Cuando falta mi médico, en el centro se preocupan de darme la atención que necesito & $0,321 *$ \\
\hline $\begin{array}{l}\text { Ante situaciones extraordinarias (ausencia de médico, paro etc.) me avisan con } \\
\text { anterioridad }\end{array}$ & $0,316^{*}$ \\
\hline
\end{tabular}

* Las correlaciones son significativas al 0,01. La escala de CCAS utilizada para este análisis se calculó como el promedio de las cinco subescalas con sus respectivos indicadores que permanecieron después del análisis factorial confirmatorio. 


\section{Discusión}

De acuerdo a los resultados de este estudio, se puede concluir, que la escala propuesta para medir la Calidad del Servicio de los centros de salud de atención secundaria, desde la perspectiva de los pacientes, presenta un buen grado de fiabilidad, validez y dimensionalidad. Esto es especialmente relevante, considerando que actualmente en los centros de atención secundaria de salud, no se han desarrollado escalas de calidad del servicio que cumplan estas condiciones.

Es necesario tener presente, que el objetivo de este estudio, fue proponer una escala de medida que cumpla con estas condiciones básicas, para que pueda ser utilizada mas adelante por los centros de atención secundarios, y no, el mostrar los resultados de su aplicación, en cuanto al nivel de calidad del servicio que cada uno de estos centros presenta.

La escala finalmente propuesta quedó constituida por cinco subescalas claramente relacionadas (confiabilidad, empatía, capacidad de respuesta, accesibilidad y tangibles) e integradas en un único constructo. Demostrando de esta manera, el carácter multidimensional y latente de este concepto, en el contexto de los centros de atención secundaria (ver anexo).

Esto plantea importantes desafíos para este tipo de centros de atención de salud, ya que a la hora de planificar y gestionar la calidad del servicio, deberían considerar estos diversos factores de forma simultánea. Esto resulta fundamental en el contexto de la gestión de las expectativas y la satisfacción de los pacientes. De hecho, al contar con una escala de medida que esté compuesta por las dimensiones claves, y además sea fiable, válida y muestre cohesión en cada uno de sus componentes, permite a estos centros de atención, medir la calidad del servicio que ofrecen, compararla con la de otros centros, tomar las acciones correctivas y generar las estrategias necesarias para entregar un servicio, que acorde a las expectativas, logre una mayor satisfacción de sus pacientes.

Si bien la salud pública chilena ha mostrado importantes resultados desde el punto de vista sanitario, la opinión pública y los medios de comunicación han dado cuenta de una atención que no responde a los estándares y expectativas de la población. Pero no desde una perspectiva de la cobertura o los resultados sanitarios, sino que desde el punto de vista de la calidad del servicio de atención. Es decir, del trato entregado a los pacientes, los tiempos de espera, la falta de información y el déficit de infraestructura, entre otros.

En este contexto, es que el Ministerio de Salud se ha planteado como uno de sus principales objetivos para el período 2000-2010: "Proveer servicios acorde a las expectativas de la población" (Ministerio de Salud, 2002), lo que implica necesariamente, conocer la calidad del servicio que se está ofreciendo. 
Para esto, lo que se requiere en concreto, es estandarizar la medición de la calidad del servicio entre los centros de atención y realizar un monitoreo regular de ella. Para lo cual pueden contar con el instrumento de medida propuesto en este estudio. Además, se deben identificar acciones tendientes a mejorar la calidad en aquellas variables, que a juicio de los usuarios del sistema, resulten deficientes. El desafío final, implica que los gestores de los centro de atención para los cuales se desarrolló esta escala de medición, avancen en la calidad de la atención como objetivo principal, y logren finalmente, que se produzcan los cambios y las inversiones necesarias, para que a través de la calidad de la atención, se logre la satisfacción de sus pacientes.

\section{Referencias bibliográficas}

ANDERSON, F. C.; GERBING, D. W. Structural equation modeling in practice: a review and recommended two-step approach. Psychological Bulletin, v. 103, n. 3, p. 411-423, 1988.

BAGOZZI, R. Evaluating structural equations models with unobservable variables and measurement error: a comment. Journal of Marketing Research, v. 18, p. 375$381,1981$.

; YI, Y. On the evaluation of structural equation models. Journal of Marketing Science, v. 6, n. 1, p. 74-94, 1988.

BOWERS, M. R.; SWAN, J. E.; KOEHLER, W. F. What attributes determine quality and satisfaction with health care delivery? Health Care Manage Review, v. 19, p. 49-55, 1994.

CHANG, C. S. et al. Customer satisfaction in medical service encounters - a comparison between obstetrics and gynecology patients and general medical patients. Journal of Nursing Research, v. 14, n. 1, p. 9- 23, 2006.

DE MAN, S. et al. Patient's and personnel's perceptions of service quality and patient satisfaction in nuclear medicine. European Journal of Nuclear Medicine and Molecular Imaging, v. 29, n. 9, p. 1109-1117, 2002.

DENG, S.; DART, J. Measuring market orientation: a multi-factor, multi-item approach. Journal of Marketing Management, v. 10, p. 725-742, 1994.

DE WULF, K.; ODEKERKEN-SCHRÖDER, G. Assessing the impact of a retailer's relationship efforts on consumer' attitudes and behavior. Journal of Retailing and Consumer Services, v. 10, n. 2, p. 95-108, 2003. 
DONABEDIAM, A. La dimensión internacional de la evaluación y la garantía de calidad. Salud Pública Méx, v. 32, n. 2, p. 113-117, 1990.

GEA, M. T. et al. Opinión de los usuarios sobre la calidad del Servicio de Urgencias del Centro Médico-Quirúrgico del Hospital Virgen de las Nieves. Revista Calidad Asistencial, v. 16, p. 37-44, 2001.

HAIR JR., J. F. . Multivariate data analysis. 5. ed. NJ: Prentice Hall, 1998.

JÖRESKOG, K. G.; SÖRBOM, D. New feature in Lisrel 8. Chicago: Scientific Software, 1993.

JUN, M.; PETERSON, R. T.; ZSIDISIN, G. A. The identification and measurement of quality dimensions in health care: focus group interview results. Health Care Manage Review, v. 23, p. 81-96, 1998.

LEWIS, J. R. Patient views on quality care in general practice: literature review. Society Science Medical, v. 39, p. 655-670, 1994.

MADERUELO, J. A. et al. Factores de insatisfacción con el sistema sanitario. Un estudio Delphi. Atención Primaria, v. 17, n. 5, p. 348-353, 1996.

MCKINLEY, R. K. et al. Reliability and validity of a new measure of patient satisfaction without of ours primary medical care in the United Kingdom: development of a patient questionnaire. BMJ Journals, v. 314, n. 18, p. 193-198, 1997.

MINISTERIO DE SALUD. Objetivos Sanitarios para la década 2000-2010. El Vigía, V. 5, n. 15, p. 1-12, 2002.

MIRA, J. J. et al. ¿Qué hace que los pacientes estén satisfechos? Análisis de la opinión de pacientes y profesionales mediante la técnica Delphi. Revista Calidad Asistencial, v. 14, p. 165-178, 1999.

et al. Evolución de la calidad percibida por los pacientes en dos hospitales públicos. Revista Psicothema, v. 3, n. 4, p. 581-585, 2001.

NATHORST-BÖÖS, J. et al. An evaluation of the QSP and the QPP: two methods for measuring patient satisfaction. International Journal Quality Health Care, v. 13, p. 257-264, 2001.

NELSON, E. C. et al. The patient judgments system: reliability and validity. $Q R B$ (Qual Rev Bull), v. 15, p. 185-191, 1989.

NUNNALLY, J. C. Psychometric. 2. ed. New York: McGraw-Hill, 1978.

NUROSIS, M. J. SPSS. Statistical data analysis. SPSS Inc., 1993.

PARASURAMAN, A.; ZEITHALM, V.; BERRY, L. Servqual: a multiple-item scale for measuring customer perceptions of service quality. Journal of Retailing, v. 64, p. 12-40, Spring 1988. 
STEENKAMP, J.; VAN TRIJP, H. The use Lisrel in validating marketing constructs. International Journal of Research in Marketing, v. 8, p. 283-299, 1991.

WARE, J.; SNYDER, M. Dimensions of patient attitudes regarding doctors and medical care services. Medical Care, v. 13, p. 669-682, 1975.

\section{Anexo}

\section{Escala de medida de calidad del servicio de los Centros de Atención Secundaría}

\section{Confiabilidad}

Cuando recibo atención en el centro, no necesito volver una segunda vez por causas de una mala atención previa

CONF1

Generalmente se observa coordinación dentro del centro

CONF2

Alguna vez han extraviado parte de mi ficha médica

CONF3**

Los resultados de exámenes de laboratorio y radiológicos realizados en el centro, siempre están ubicables y son confiables

CONF4**

Los profesionales del centro, ejecutan de buena forma sus tareas, son

competentes

CONF5

\section{Empatía}

El personal del centro, en general conoce mis intereses (gustos y preferencias)

$\mathrm{EMP} 1^{* *}$

El personal del centro, en general comprende mis necesidades

EMP2

El personal del centro, en general me trata con cortesía y respeto

EMP3

El personal del centro, en general me trata de manera correcta. Por ejemplo:

explicaciones, forma de hablar

EMP4

La atención y explicación de los médicos en general es buena

EMP5

La atención de las enfermeras y técnicos paramédicos en general es buena

EMP6

La atención del personal administrativo (ventanillas) en general es buena

EMP7

\section{Capacidad de respuesta}

Las especialidades que se otorgan son suficientes

Siempre que lo necesito, tengo una hora de acuerdo con la urgencia con que fue solicitada

Espero poco tiempo desde la hora de mi cita hasta entrar en la consulta del médico especialista 


\section{Accesibilidad}

Por lo general, tengo acceso a toda la información existente de mi estado de salud

$\mathrm{ACC} 1$

Siempre que lo necesito, tengo acceso a todas las especialidades médicas

Siempre que lo necesito, tengo acceso a todos los exámenes que se pueden realizar

en este centro

ACC3

En el centro, en general, atienden mis consultas y resuelven todas mis dudas

ACC4

Siempre tengo acceso a escoger mi médico tratante

ACC5*

\section{Tangibles}

El mobiliario de la sala de espera y del box médico es cómodo y confortable

TAN 1

Creo que la tecnología que hay en el Centro Asistencial, es la adecuada TAN2

La limpieza del centro es la adecuada

TAN3

Las salas de espera se encuentran en condiciones adecuadas (por ejemplo, temperatura y ruidos)

TAN4

Los box médicos se encuentran en condiciones adecuadas (por ejemplo, temperatura y ruidos)

TAN5

Los baños del centro se encuentran en condiciones adecuadas (por ejemplo, limpieza y ventilación)

El acceso al centro es adecuado (por ejemplo, entradas y locomoción)

* Ítem eliminado en el primer proceso de depuración.

** Ítems eliminados en el tercer proceso de depuración. 